TITLE:

\title{
Short-time dynamics and magnetic critical behavior of the two- dimensional random-bond Potts model
}

\section{$\operatorname{AUTHOR}(\mathrm{S}):$}

Ying, He-Ping; Harada, Kenji

\section{CITATION:}

Ying, He-Ping ... [et al]. Short-time dynamics and magnetic critical behavior of the twodimensional random-bond Potts model. Physical Review E 2000, 62(1): 174-178

\section{ISSUE DATE:}

2000-07-01

URL:

http://hdl.handle.net/2433/200796

RIGHT:

(C)2000 The American Physical Society 


\title{
Short-time dynamics and magnetic critical behavior of the two-dimensional random-bond Potts model
}

\author{
He-Ping Ying \\ Center for Computational Physics, University of Tsukuba, Tsukuba, Ibaraki 305-8577, Japan, \\ and Zhejiang Institute of Modern Physics, Zhejiang University, Hangzhou 310027, People's Republic of China \\ Kenji Harada \\ Department of Applied Analysis and Complex Dynamical Systems, Kyoto University, Kyoto 606-8501, Japan
}

(Received 21 January 2000)

\begin{abstract}
The critical behavior in the short-time dynamics for the random-bond Potts ferromagnet in two dimensions is investigated by short-time dynamic Monte Carlo simulations. The numerical calculations show that this dynamic approach can be applied efficiently to study the scaling characteristic, which is used to estimate the critical exponents $\theta, \beta / \nu$, and $z$, for quenched disordered systems from the power-law behavior of the $k$ th moments of magnetization.
\end{abstract}

PACS number(s): $64.60 . \mathrm{Fr}, 05.50 .+\mathrm{q}, 75.40 . \mathrm{Mg}, 64.60 . \mathrm{Ht}$

\section{INTRODUCTION}

An understanding of the effects of quenched impurities on the nature of phase transitions is one of the significant subjects in statistical physics, and it has been a topic of substantial interest for many authors in the last two decades [1-9]. According to the Harris criterion [11], quenched randomness is a relevant perturbation at the second-order critical point when the specific-heat exponent $\alpha$ of the pure system is positive. Following the earlier work of Imry and Wortis [1], who argued that a quenched disorder could produce rounding of a first-order phase transition and thus induce second-order phase transitions, the introduction of randomness to systems undergoing a first-order phase transition has been comprehensively considered. It was shown by Hui and Berker with phenomenological renormalization group arguments that bond randomness can have a drastic effect on the nature of a first-order phase transition [2], and the feature has been placed on a firmer basis with a rigorous proof of vanishing of the latent heat [3]. Their theory was numerically checked with the Monte Carlo (MC) method by Chen, Ferrenberg and Landau (CFL) [4,5], who studied the eight-state Potts model with random-bond disorder. Experimental evidence has been found in two-dimensional systems that in the order-disorder phase transitions of absorbed atomic layers, the critical exponents are modified, on the addition of disorder, from the original four-state Potts model universality class in the pure case $[12,13]$. On the other hand, no modification is found when the pure system belongs to the Ising universality class [14]. The theoretical study of such disordered systems is also an active field where use of intensive MC simulations is often helpful [10,15-19].

It is well known that the pure Potts model in two dimensions (2D) has a second-order phase transition when the number of Potts states $q \leqslant 4$ and is first order for $q>4$. As the specific-heat exponent $\alpha$ of the pure system is always positive for $q>2$, the disorder will be the relevant perturbation for the Potts model. As a result, all the transitions are second order for the 2D $q$-state Potts models in the presence of quenched disorder, and the impurities have a particularly strong effect for $q>4$, even changing the order of the transitions.

In this paper, we discuss the dynamic scaling features of the random-bond Potts model (RBPM) through MC simulations, to estimate the critical exponents. We consider the important questions of whether there exists an Ising-like universality class for the RBPM and how the critical behavior is affected by the introduction of disorder into the pure system [17]. The large-scale MC results of CFL and in Ref. [18] suggest that, in $2 \mathrm{D}$, any random system should belong to the pure Ising universality class. These results are also consistent with experiments [12]. In recent papers [7,8], however, Cardy and Jacobsen studied the random-bond Potts models for several values of $q$ with a different approach based on the connectivity transfer matrix (TM) formalism of Blöte and Nightingale [20]. Their estimates of the critical exponents lead to a continuous variation of $\beta / \nu$ with $q$, which is in sharp disagreement with the MC results for $q=8[4,5]$. We hope that the resulting critical behavior measured in this paper will play a role in settling this controversy. Furthermore, we will test the short-time dynamic (STD) MC approach to study spin systems with quenched disorder and show its efficiency with numerical studies, which is also one of the main aims of this paper.

\section{MODEL AND METHOD}

The Hamiltonian of the $q$-state Potts model with quenched random interactions can be written as

$$
-\beta H=\sum_{\langle i, j\rangle} K_{i j} \delta_{\sigma_{i} \sigma_{j}}, \quad K_{i j}>0,
$$

where the spin $\sigma$ can take the values $1, \ldots, q, \beta=1 / k_{B} T$ is the inverse temperature, $\delta$ is the Kronecker delta function, and the sum is over all nearest-neighbor pairs on a 2D lattice. The dimensionless couplings $K_{i j}$ are selected from two positive (ferromagnetic) values of $K_{1}$ and $K_{2}=r K_{1}$, with a 
strong-to-weak coupling ratio $r=K_{2} / K_{1}$ called the disorder amplitude, according to a bimodal distribution,

$$
P(K)=p \delta\left(K-K_{1}\right)+(1-p) \delta\left(K-K_{2}\right) .
$$

When $p=0.5$, the system is self-dual and the exact critical point can be determined from [21]

$$
\left(e^{K_{c}}-1\right)\left(e^{K_{c}^{\prime}}-1\right)=q,
$$

where $K_{c}$ and $K_{c}^{\prime}$ are the critical values of $K_{1}$ and $K_{2}$, respectively, at the transition point. $r=1$ corresponds to the pure case, the critical point is located at $K_{c}=\ln (1+\sqrt{q})$, and the phase transitions are first order for $q>4$. With additional random-bond distribution, however, new second-order phase transitions are induced for any $q$-state Potts model and the new critical points are determined according to Eq. (3) for different values of disorder amplitude $r$ and state parameter $q$.

In this work we chose $q=8$, which is known to have a strong first-order phase transition, in the hope that we would find a new second-order phase transition caused by the quenched disorder to show the effect of impurities on the first-order system. Several values of $r$ were used, as in $[5,6,10]$, to check the Ising-like universality class. To minimize the number of bond configurations needed for the disorder averages, we confined our study to the bond distributions in which there are the same number of strong and weak bonds in each of the two lattice directions. This procedure should reduce the variation between different bond configurations, with no loss of generality.

We performed our simulations by the STD method [22] on 2D square lattices with periodic boundary conditions. These dynamic MC simulations have been successfully performed to estimate the critical temperatures $T_{c}$ and the critical exponents $\theta, \beta, \nu$, and dynamic exponent $z$ for the 2D Ising model [23] and the 2D three-state Potts model [24], since for both models there exist second-order phase transitions. Recently this approach has also been extensively applied to the Fully frustrated $X Y$ model and spin glass systems to study the critical scaling characteristic, which is used to estimate all the dynamic and static critical exponents [2527].

Traditionally, it was believed that universality and scaling relations could be found only in the equilibrium stage or long-time regime. In Ref. [28], however, it was discovered that for a magnetic system in states with a very high temperature $T \gg T_{c}$ suddenly quenched to the critical temperature $T_{c}$ and then evolving according to a dynamics of model $A$ [29], there emerges a universal dynamic scaling behavior already within the short-time regime, which satisfies

$$
M^{(k)}\left(t, \tau, L, m_{0}\right)=b^{-k \beta / \nu} M^{(k)}\left(b^{-z} t, b^{1 / \nu} \tau, b^{-1} L, b^{x_{0}} m_{0}\right),
$$

where $M^{(k)}$ is the $k$ th moment of the magnetization, $\tau=(T$ $\left.-T_{c}\right) / T_{c}$ is the reduced temperature, $\beta$ and $\nu$ are the well known static critical exponents, and $b$ is a scaling factor. The variable $x_{0}$, a new independent exponent, is the scaling dimension of the initial magnetization $m_{0}$. This dynamic scaling form is generalized from finite size scaling in the equilibrium stages [33]. It is important to note that the scaling behavior of Eq. (4) can be applied to both dynamic exponent measurements and estimates of the static exponents originally defined in equilibrium.

We begin our study of the evolution of magnetization in the initial stage of the dynamic relaxation starting at very high temperature and small magnetization $\left(m_{0} \sim 0\right)$. For a sufficiently large lattice $(L \rightarrow \infty)$, from Eq. (4) by setting $\tau$ $=0, b=t^{1 / z}$, it is easy to derive that

$$
M^{(k)}\left(t, m_{0}\right)=t^{-k \beta / \nu z} M^{(k)}\left(1, t^{x_{0} / z} m_{0}\right) .
$$

When $k=1$ we get the most important scaling relation on which our measurements of the critical exponent $\theta$ are based,

$$
M(t) \sim m_{0} t^{\theta}, \quad \theta=\left(x_{0}-\beta / \nu\right) / z .
$$

As a result, the magnetization undergoes an initial increase at the critical point $K_{c}$ after a microscopic time $t_{m i c}$. This prediction is supported by a number of MC investigations which have been applied to detect all the static and dynamic critical exponents [23,24] as well as the critical temperatures $[25,31]$. The advantage of the dynamic MC simulation is that it may eliminate critical slowing down, since the measurements are performed in the early time stages of the evolution where the spatial and time correlation lengths are small.

In our simulations, the time evolution of $M(t)$ is calculated through the definition

$$
M(t)=\frac{1}{N}\left[\frac{q\left\langle M_{O}\right\rangle-N}{q-1}\right] .
$$

Here $M_{O}=\max \left(M_{1}, M_{2}, \ldots, M_{q}\right)$ with $M_{i}$ being the number of spins in the $i$ th state among $q$ states. $\langle\cdots\rangle$ denotes the initial configuration averages over independent random number sequences, and $[\cdots]$ the disorder configuration averages over quenched random-bond distributions. $N=L^{2}$ is the number of spins on this square lattice and $q=8$ is chosen.

The susceptibility plays an important role in the equilibrium. Its finite size behavior is often used to determine the critical temperature and the critical exponents $\gamma / \nu$ and $\beta / \nu$ [5]. For the STD approach, the time-dependent susceptibility (the second moment of the magnetization) is also interesting and important. For the random-bond Potts model, the second moment of the magnetization is usually defined as

$$
M^{(2)}(t)=\frac{1}{N}\left[\left\langle M^{2}(t)\right\rangle-\langle M(t)\rangle^{2}\right] .
$$

To study the scaling behavior of the second moment of magnetization, we have to take the initial states of $m_{0}=0$ to start the relaxation processes. Because the spatial correlation length at the beginning of the relaxation is small compared with the lattice size $L^{d}$ in the short-time regime of the dynamic evolution, the second moment behaves as $M^{(2)}(t, L)$ $\sim L^{-d}$. Then the finite size scaling Eq. (4) induces a powerlaw behavior at the critical temperature,

$$
M^{(2)}(t) \sim t^{y}, \quad y=(d-2 \beta / \nu) / z .
$$

From a scaling analysis of the spatial correlation function we easily realize the nonequilibrium spatial correlation length $\xi \sim t^{1 / z}$. Therefore $M^{(2)}(t) \sim \xi^{(d-2 \beta / \nu)}$. 
TABLE I. The tendency and measured values of $\theta$ as a function of the disorder amplitude $r$ for different initial $m_{0}$ at the critical points $K_{c}$ on a $64^{2}$ lattice.

\begin{tabular}{lcccccc}
\hline \hline \multicolumn{7}{c}{$m_{0}$} \\
$r$ & 0.06 & 0.04 & 0.02 & 0.01 & $\theta$ & $K_{c}(r)$ \\
\hline 2 & $0.310(8)$ & $0.338(8)$ & $0.350(8)$ & $0.352(7)$ & $0.353(6)$ & $0.920185271 \ldots$ \\
5 & $0.160(6)$ & $0.215(6)$ & $0.252(5)$ & $0.257(4)$ & $0.262(4)$ & $0.512307010 \ldots$ \\
8 & $0.106(5)$ & $0.167(4)$ & $0.208(4)$ & $0.216(3)$ & $0.221(3)$ & $0.367963156 \ldots$ \\
10 & $0.090(4)$ & $0.146(3)$ & $0.193(3)$ & $0.201(3)$ & $0.203(3)$ & $0.312655667 \ldots$ \\
\hline
\end{tabular}

In the above considerations the dynamic relaxation process was assumed to start from a disordered state or with small magnetization $m_{0}$. Another interesting and important process is dynamic relaxation from a completely ordered state. The initial magnetization is located exactly at its fixed point $m_{0}=1$, where scaling of the form

$$
M^{(k)}(t, \tau, L)=b^{-k \beta / \nu} M^{(k)}\left(b^{-z} t, b^{1 / \nu} \tau, b^{-1} L\right)
$$

is expected [30]. This scaling form appears the same as the dynamic scaling one in the long-time regime; however, it is now assumed to be already valid in the macroscopic shorttime regime. For the magnetization itself, $b=t^{1 / z}$ yields, for a sufficiently large lattice,

$$
M(t, \tau)=t^{-\beta / \nu z} M\left(1, t^{-\beta / \nu z} \tau\right) .
$$

This leads to a power-law decay behavior of

$$
M(t, \tau)=t^{-c_{1}}, \quad c_{1}=\beta / \nu z,
$$

at the critical point $(\tau=0)$. The formula can be used to calculate the critical exponents $\beta / \nu$ and $z$. For a small but nonzero $\tau$, the power-law behavior will be modified by the scaling function $M\left(1, t^{-\beta / \nu z} \tau\right)$, which has been used to determine the critical temperatures $[31,32]$. Furthermore, by introducing a Binder cumulant

$$
U(t, L)=\frac{M^{(2)}(t, L)}{[M(t, L)]^{2}}-1,
$$

a similar power-law behavior at the critical point induced from the scaling Eq. (10) shows that

$$
U(t, L) \sim t^{c_{2}}, \quad c_{2}=d / z,
$$

on a large enough lattice. Here, unlike the relaxation from the disordered state, the fluctuations caused by the initial configurations are much smaller. In practical simulations, these measurements of the critical exponents and critical temperature are better in quality than those from the realization process starting from disordered states.

\section{MC SIMULATIONS AND RESULTS}

Since it has been pointed out that the heat-bath algorithm is more efficient than the Metropolis algorithm in the STD [24], and universality is satisfied for different algorithms, we perform the MC simulations only with the heat-bath algorithm at the critical points of a 2D eight-state RBPM for an optimal disorder amplitude $r^{*}=10$, which is located in the random fixed point regime with the largest value of central charge $c=1.5300(5)$ [10]. Samples for averages are taken over both 300 disorder distribution configurations and about 500 independent initial configurations on the square lattices $L^{2}$ with $L$ up to 128 . Statistical errors are simply estimated by performing three groups of averages with different random seed selects for the initial configurations. It should be noted that, except for $M(t)$, the measurements of $M^{(2)}(t)$ and $U(t)$ are restricted to initial states with $m_{0}=0$ or $m_{0}$ $=1$. It was verified that the critical exponents have the same values as those in the equilibrium or long-time stage of the relaxation [23]. Therefore we can measure these exponents based on the corresponding scaling relation in the initial stages of the relaxation.

We start our simulations to verify the power-law scaling behavior of $M(t)$ with several values of disorder amplitude at the critical points $K_{c}(r)$ shown in Table I. The initial configurations are prepared with small magnetization $m_{0}$ $=0.06-0.01$ and exact zero states. In Fig. 1, the time evolution of the magnetization $M(t)$ versus the disorder amplitude $r$ on a $64^{2}$ lattice is displayed with a double-logarithmic scale. We can easily find that all the curves exhibit the power-law behavior predicted by Eq. (6). Thus $\theta$ can be estimated from the slopes of the curves. The values of $\theta$ as a function of the disorder amplitude $r$ for small initial magnetization $m_{0}$ are presented in Table I.

We then set $m_{0}=0$ to measure the second moment of magnetization. Power-law behavior of the second moment $M^{(2)}(t)$ is observed in Fig. 2, where the curves for different

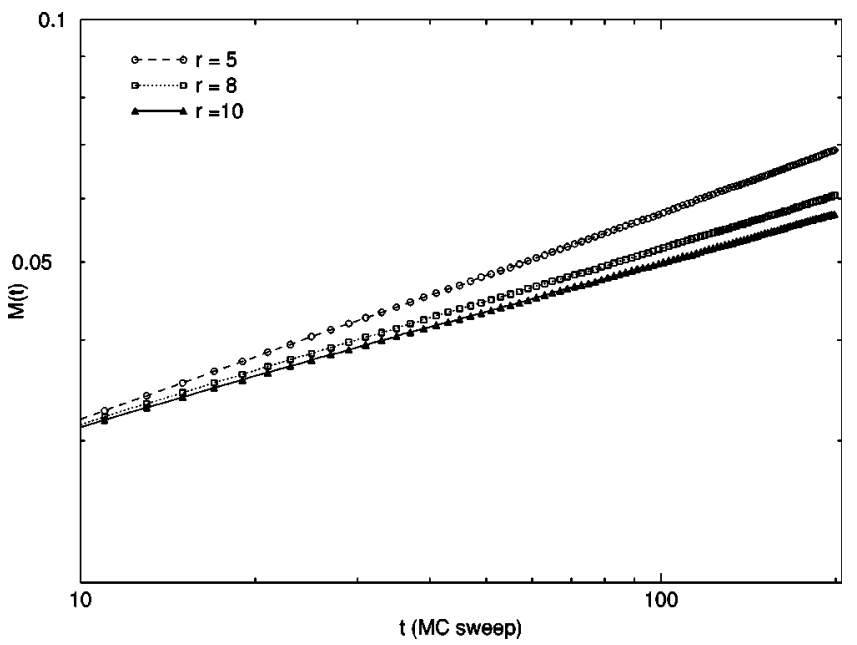

FIG. 1. The time evolution of magnetization showing the $r$ dependence of $\theta$, plotted on a double-logarithmic scale on a lattice of $64 \times 64$ with $m_{0}=0.01$. 


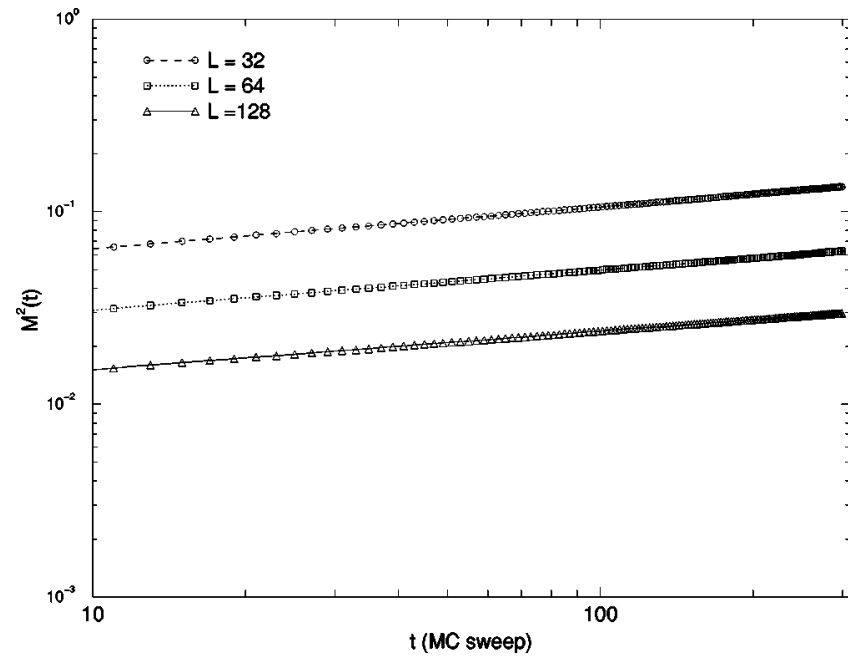

FIG. 2. The time evolution of the second moment of magnetization starting from absolute random states, plotted on a doublelogarithmic scale on lattices of $32 \times 32,64 \times 64$, and $128 \times 128$.

lattice sizes are plotted. Again, they present a very nice power-law increase. Values of scaling dimension $y=(d$ $-2 \beta / \nu) / z$ determined from slopes of the curves during $t$ $=[10,200]$ are listed in Table II.

We further set $m_{0}=1$ to observe the evolution of the magnetization and the Binder cumulant; both should show power-law behavior as predicted by Eq. (12) and Eq. (14). Their curves are plotted in Figs. 3 and 4, respectively. The values of the scaling dimensions $c_{1}=\beta / \nu z$ and $c_{2}=d / z$ were then estimated and are shown in Table II. Now the results for $y, c_{1}=\beta / \nu z$, and $c_{2}=d / z$ can be used to estimate the critical exponent $\beta / \nu$, which is also presented in Table II. For comparison, also listed in Table II are the corresponding results for the scaling dimension for the Ising and $q=3$ Potts models on 2D (3D) square (cubic) lattices, and in Table III we summarize the results for the critical exponent $\beta / \nu$ up to the present.

\section{SUMMARY AND CONCLUSION}

In this paper we have investigated the short-time critical dynamics of the random-bond Potts model on 2D lattices to verify whether it has a second-order phase transition in the Ising-like universality class. Dynamic scaling behavior was found, and has been used to estimate the critical exponents $\theta, z$, and $\beta / \nu$. Our main results are summarized in Table II; they are obtained from the slopes of power-law curves for

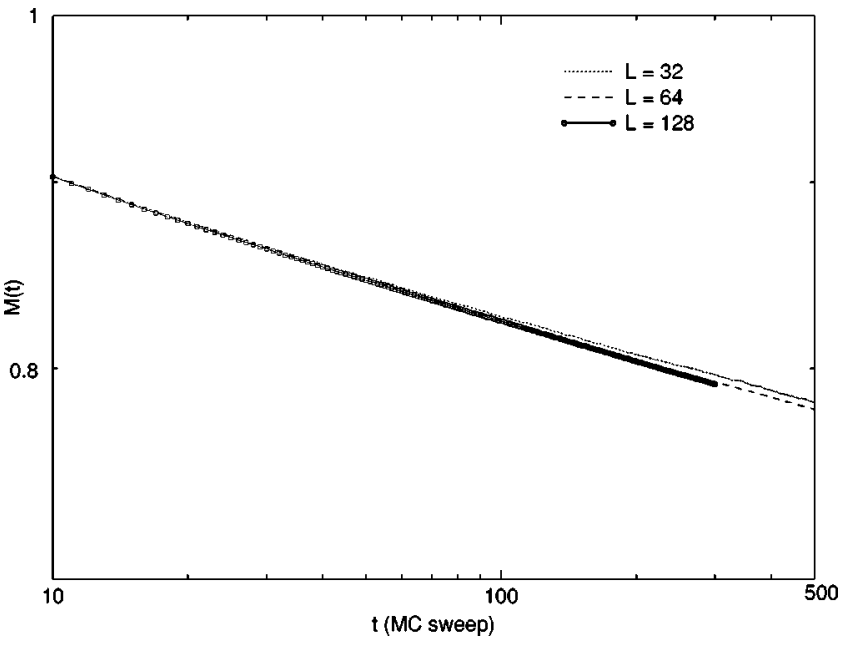

FIG. 3. The power-law decay of magnetization starting from fully ordered states, plotted on a double-logarithmic scale on lattices of $32 \times 32,64 \times 64$, and $128 \times 128$. The finite size effect is obvious when the lattice size $L<64$.

$M(t), M^{(2)}(t)$, and $U(t)$ on double-logarithmic scales by least squares fits.

Our work shows that for the RBPM there exists a powerlaw behavior, which is a typical feature of a continuous phase transition in STD processes. The $r$ dependence characteristics of the dynamic exponent $\theta$ gives evidence that the dynamic MC behavior is different from that of the pure Ising model, and the values of the magnetic exponent $\beta / \nu$ in our calculation seem to be the same as those given by the TM formula [8], but not those by given by CFL. For the apparent $m_{0}$ dependence of $\theta$ we argue that the effect of $\theta=\left(x_{0}\right.$ $-\beta / \nu) / z$ on the initial magnetization $m_{0}$ is due to the fact that the scaling dimension $x_{0}$ is determined by a critical characteristic function $\chi\left(b, m_{0}\right)=b^{x\left(b, m_{0}\right)} m_{0}$, which is a nontrivial function of $m_{0}$ and shows an off-fixed-point correction for the exponent $\theta$ when $m_{0}$ deviates from the fixed point of $m_{0}=0$ [34].

In conclusion, this study presents numerical evidence that the quenched impurities in the RBPM can induce secondorder phase transitions, but they appear not always to belong to the Ising-like universality class, although the result for the critical exponent $\theta$ is the same for both the $r=10 \mathrm{RBPM}$ and the Ising model within the error bars by present calculations. Second, as the effect of critical slowing down in the equilibrium stage for the RBPM is more severe than that for pure systems, cluster algorithms have been frequently used up to

TABLE II. The values of scaling exponents for the 2D $q=8$ RBPM with $r=10$, measured from the scaling functions $M(t), M^{(2)}(t)$, and $U(t)$, respectively, starting from both random initial states and ordered states. Also listed are those for the 2D Ising and $q=3$ Potts models, and the 3D Ising model [22,24,32].

\begin{tabular}{|c|c|c|c|c|c|}
\hline Exponent & $m_{0}$ & 2D RBPM & 2D Ising & 2D Potts & 3D Ising \\
\hline$\theta$ & $\sim 0.0$ & $0.197(4)$ & $0.191(1)$ & $0.075(3)$ & $0.108(2)$ \\
\hline$y=(d-2 \beta / \nu) / z$ & & $0.438(6)$ & $0.817(7)$ & $0.788(1)$ & \\
\hline$c_{1}=\beta / \nu z$ & $=1.0$ & $0.0390(6)$ & $0.056(1)$ & $0.065(1)$ & $0.2533(7)$ \\
\hline$c_{2}=d / z$ & & $0.518(9)$ & $0.926(8)$ & $0.934(9)$ & $0.1462(12)$ \\
\hline $2 \beta / \nu=d-y z$ & & $0.302(6)$ & $0.240(36)$ & $0.269(7)$ & $1.034(4)$ \\
\hline $2 \beta / \nu($ exact $)$ & & & $1 / 4$ & $4 / 15$ & \\
\hline
\end{tabular}




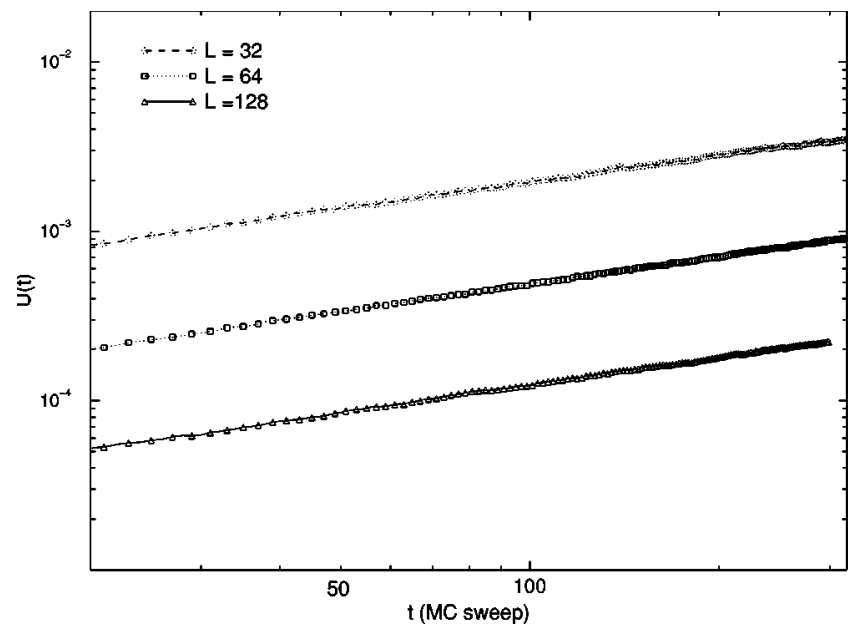

FIG. 4. The time evolution of the Binder cumulant starting from fully ordered states, plotted on a double-logarithmic scale on lattices of $32 \times 32,64 \times 64$, and $128 \times 128$.

now in MC simulations of the 2D RBPM $[4,5,9,10,19]$. In the present paper, however, we have applied STD MC simulations, which use local updating schemes for the 2D RBPM. The fact that dynamic MC simulations can avoid the critical slowing down in STD processes, where the spatial correlation length is still small, makes it easier to calculate the critical exponents. An important subject for further study is
TABLE III. Results for the magnetic scaling exponent $\beta / \nu$ estimated by different methods for the 2D eight-state RBPM.

\begin{tabular}{lccc}
\hline \hline \multicolumn{1}{c}{ Reference } & $r$ & $\beta / \nu$ & Technique \\
\hline CFL [4] & 2 & $0.118(2)$ & MC \\
Cardy and Jacobsen [8] & 2 & $0.142(4)$ & TM \\
Chatelain and Berche [9] & 10 & $0.153(3)$ & MC \\
Olson and Young [17] & & $0.156(3)$ & MC \\
Picco [35] & 10 & $0.153(1)$ & MC \\
Present work & 10 & $0.151(3)$ & STD \\
\hline \hline
\end{tabular}

the dependence of the dynamic critical exponent $\beta / \nu$ on the state parameter $q$ and the disorder amplitude $r$ by systematic simulations using the STD method in order to clarify the crossover behavior from the random fixed point to a percolationlike limit [35]. This is being studied at present.

\section{ACKNOWLEDGMENTS}

We acknowledge helpful discussions with Y. Aoki and $\mathrm{H}$. P. Shanahan. This research was initiated during a visit to the University of Tsukuba by H.P.Y., who also acknowledges the hospitality of the Center for Computational Physics, where the MC simulations were performed on DEC workstations. The work of H.P.Y. has been supported in part by the NNSF of China under Grant No. 19975041.
[1] Y. Imry and M. Wortis, Phys. Rev. B 19, 3580 (1979).

[2] K. Hui and A.N. Berker, Phys. Rev. Lett. 62, 2507 (1989).

[3] M. Aizenman and J. Wehr, Phys. Rev. Lett. 62, 2503 (1989).

[4] S. Chen, A.F. Ferrenberg, and D.P. Landau, Phys. Rev. Lett. 69, 1213 (1992).

[5] S. Chen, A.F. Ferrenberg, and D.P. Landau, Phys. Rev. E 52, 1377 (1995).

[6] M. Picco, Phys. Rev. B 54, 14930 (1996); Phys. Rev. Lett. 79, 2998 (1997).

[7] J. Cardy, J. Phys. A 29, 1897 (1996).

[8] J. Cardy and J.L. Jacobsen, Phys. Rev. Lett. 79, 4063 (1997).

[9] C. Chatelain and B. Berche, Phys. Rev. Lett. 80, 1670 (1998).

[10] C. Chatelain and B. Berche, Phys. Rev. E 58, R6899 (1998); 60, 3853 (1999).

[11] A.B. Harris, J. Phys. C 7, 129 (1974).

[12] L. Schwenger, K. Buddle, C. Voges, and H. Pfnür, Phys. Rev. Lett. 73, 296 (1994).

[13] C. Voges and H. Pfnür, Phys. Rev. B 57, 3345 (1998).

[14] Ch.V. Mohan, H. Kronmüller, and M. Kelsch, Phys. Rev. B 57, 2701 (1998).

[15] S. Wiseman and E. Domany, Phys. Rev. E 51, 3074 (1995).

[16] J.-K. Kim, Phys. Rev. B 53, 3388 (1996).

[17] T. Olson and A.P. Young, Phys. Rev. B 60, 3328 (1999), and references therein.

[18] M. Kardar, A.L. Stella, G. Sartoni, and B. Derrida, Phys. Rev. E 52, R1269 (1995).
[19] F. Yasar, Y. Gündüc, and T. Celik, Phys. Rev. E 58, 4210 (1998).

[20] H.W.J. Blöte and M.P. Nightingale, Physica A 112, 405 (1982).

[21] W. Kinzel and E. Domany, Phys. Rev. B 23, 3421 (1981).

[22] B. Zheng, Int. J. Mod. Phys. B 12, 1419 (1998).

[23] Z.B. Li, L. Schülke, and B. Zheng, Phys. Rev. Lett. 74, 3396 (1995); Phys. Rev. E 53, 2940 (1996).

[24] K. Okano, L. Schülke, K. Yamagishi, and B. Zheng, Nucl. Phys. B: Field Theory Stat. Syst. 485 [FS], 727 (1997).

[25] H.J. Luo, L. Schülke, and B. Zheng, Phys. Rev. Lett. 81, 180 (1998); Phys. Rev. E 57, 1327 (1998).

[26] H.J. Luo, L. Schülke, and B. Zheng, Mod. Phys. Lett. B (to be published); e-print cond-mat/9909325.

[27] H.P. Ying, H.J. Luo, L. Schülke, and B. Zheng, Mod. Phys. Lett. B 12, 1237 (1998).

[28] H.K. Janssen, B. Schaub, and B. Schmitmann, Z. Phys. B: Condens. Matter 73, 539 (1989).

[29] P.C. Hohenberg and B.I. Halperin, Rev. Mod. Phys. 49, 435 (1977).

[30] Nobuyasu Ito, Physica A 192, 604 (1993).

[31] L. Schülke and B. Zheng, Phys. Lett. A 204, 295 (1995).

[32] A. Jaster, J. Mainville, L. Schülke, and B. Zheng, J. Phys. A 32, 1395 (1999).

[33] K. Binder and D. W. Heermann, Monte Carlo Simulation in Statistical Physics (Springer, Berlin, 1992).

[34] B. Zheng, Phys. Rev. Lett. 77, 679 (1996).

[35] M. Picco, e-print cond-mat/9802092. 